\title{
Perfusion Index-Guided Management of Hypotension-Induced Arrhythmia in a Child
}

\author{
Ramón Eizaga Rebollar*1, Javier Morales Guerrero ${ }^{1}$, Luis Miguel Torres Morera $^{1}$ and María Victoria \\ García Palacios ${ }^{2}$
}

${ }^{1}$ Department of Anesthesiology and Reanimation, Puerta de Mar University Hospital, Spain

${ }^{2}$ Department of Preventive Medicine and Public Health, Puerta del mar University Hospital, Spain

Received: February 12, 2017; Published: February 21, 2018

*Corresponding author: Ramón Eizaga Rebollar, Puerta del Mar University Hospital, Ana de Viya, 21, 11009, Cádiz, Spain, Tel: 0034-956002897;

Email: ramonchueizaga@hotmail.com

\begin{abstract}
A healthy 13-year-old child underwent day-surgery excision of pilonidal sinus. After the induction, atrial bigeminy was detected in the ECG. Both heart rate (HR) and blood pressure (BP) were initially considered normal from preoperative baseline values, but not the perfusion index (PI), which seemed to reflect a low peripheral perfusion. Therefore, BP was reconsidered as inadequate and hypotension as the probable cause of the arrhythmia, and so it was treated. We believe that PI represents a valuable tool to predict intraoperative hypotension, and to guide its management.
\end{abstract}

Keywords: Perfusion index; Hypotension; Pediatric Anesthesia; Arrhythmia; Child

\section{Introduction}

Blood pressure monitoring is a standard of care in the perioperative setting which is not given enough importance in the noncardiac pediatric surgical population, despite its clinical relevance in the pediatric emergency and critical care settings.

\section{Case Description}

We present a healthy 13-year-old child, classified as ASA-1 (American Society of Anesthesiologists physical status classification system), who underwent day-surgery excision of pilonidal sinus and whose preoperative baseline BP was approximately 90/60 mmHg. Midazolam premedication and propofol-fentanyl induction were performed (maintenance with sevoflurane). Immediately after the induction and before the start of surgery, atrial bigeminy was observed in the ECG, with BP 78/35 (40) mmHg and PI of $3.24 \%$ (Figure 1A), being administered boluses of lactated Ringer's $250 \mathrm{ml}$ and ephedrine $5 \mathrm{mg}$. After two minutes, the ECG pattern changed to atrial quadrigeminy, increasing both the BP and PI to 84/35 (51) mmHg and 5.39\%, respectively (Figure 1B), being injected an additional ephedrine bolus ( $5 \mathrm{mg}$ ). Two minutes later, the ECG pattern finally changed to atrial ectopic rhythm -which persisted throughout the $30 \mathrm{~min}$ procedure-, increasing BP to 93/59 (71) $\mathrm{mmHg}$ but not PI, which remained at 5.43\% (Figure
1C). other monitored parameters were satO2 100\% (FiO2 0.4), HR 75-90 bpm, and bispectral index (BIS) 45-50.

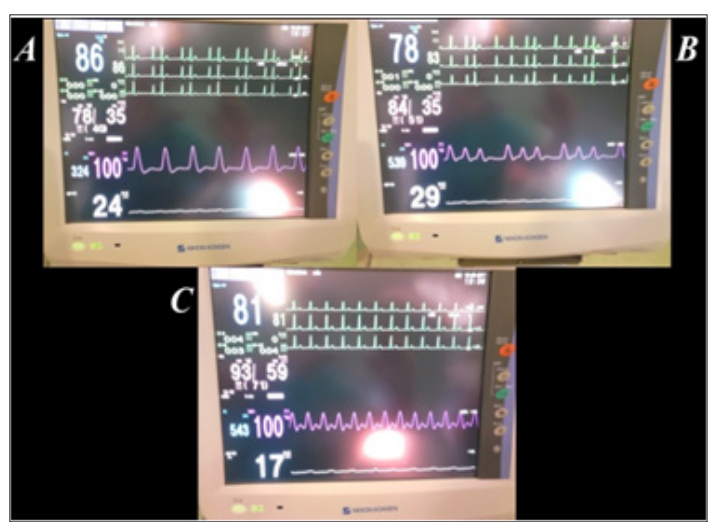

Figure 1: A. Atrial bigeminy (after propofol induction). B. Atrial quadrigeminy (after $1^{\text {st }}$ ephedrine bolus). C. Atrial ectopic rhythm (after $2^{\text {nd }}$ ephedrine bolus).

\section{Discussion}

Significant intraoperative hypotension in children has been illdefined as a $20-30 \%$ decrease from preoperative baseline BP. It is a very common event after the general anesthesia induction (over 
$30 \%$ ), especially in adolescents, propofol inductions, preoperative hypotension and high ASA status. There are few papers about anesthesia-related hypotension in healthy children and there is no clear consensus on the lower limit of normal BP, and its clinical significance or repercussion [1-4]. Anesthesia-related hypotension may associate compensatory peripheral vasoconstriction, which can result in tissue hypoperfusion. Perfusion index is the ratio of the pulsatile blood flow to the nonpulsatile or static blood flow, which can be obtained from the pulse oximeter and represents an indirect, noninvasive, continuous and early measure of peripheral perfusion and circulatory status. It may be a useful tool to predict intraoperative hypotension, and to guide its management [5].

Regarding our patient, bradycardia and hypotension were initially ruled out as causes of the arrhythmia, considering the HR normal for her age and the BP decrease normal from preoperative baseline values. However, PI was lower than 4\% (PI values seem to be generally higher than $4 \%$ when both analgosedation and circulatory status are adequate), which made us note the presence of peripheral hypoperfusion. Consequently, BP was reconsidered as inadequate and hypotension as the probable cause of the arrhythmia. Therefore, we decided to treat hypotension and arrhythmia with ephedrine, detecting a positive response in both cases when PI increased above 5\%. Thus, we highlight the importance of intraoperative BP management in relation not only to preoperative baseline values, but also and especially to other parameters of peripheral tissue perfusion, such as PI, which represents an easy and valuable tool to guide that management.

\section{References}

1. Nafiu 00, Kheterpal S, Morris M, Reynolds PI, Malviya S, et al. (2009) Incidence and risk factors for preincision hypotension in noncardiac pediatric surgical population. Paediatr Anaesth 19: 232-239.

2. Sury MR, Broadhead MW (2009) Hypotension during anesthesia before surgery. Paediatr Anaesth 19: 1037-1040.

3. Nafiu OO, Voepel-Lewis T, Morris M, Chimbira WT, Malviya S, et al. (2009) How do pediatric anesthesiologists define intraoperative hypotension? Paediatr Anaesth 19: 1048-1053.

4. Shung J (2010) Intra operative hypotension in children: does it matter? Southern African Journal of Anaesthesia and Analgesia 16: 42-44.

5. Kowalczyk M, Fijalkowska A, Nestorowicz A (2013) New generation pulse oximetry in the assessment of peripheral perfusion during general anesthesia-a comparison between propofol and desflurane. Anesthesiol Intensive Ther 45: 138-144.
(C) (i) This work is licensed under Creative

Submission Link: http://biomedres.us/submit-manuscript.php

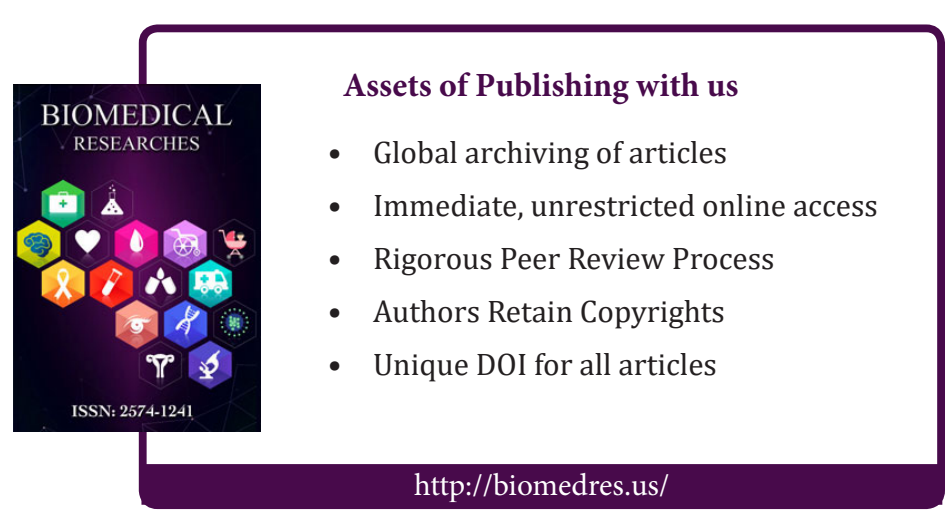

